Exp. Anim. 56(1), 21-28, 2007

\title{
Age-Dependent Changes in Iron Deposition in the Gerbil Hippocampus
}

\author{
Ki-Yeon YOO'), In Koo HWANG'), Il-Jun KANG ${ }^{2)}$, Tae-Cheon KANG'), Hak-Ju LEE), \\ Ha-Young $\mathrm{KANG}^{3)}$, Hyeon Yong $\mathrm{LEE}^{4)}$, Yang-Seok $\mathrm{OH}^{5)}$, and Moo Ho WON${ }^{1)}$ \\ 1)Department of Anatomy, College of Medicine, ${ }^{2}$ Department of Food Science and Nutrition, Hallym \\ University, Chuncheon 200-702, 3)Division of Wood Chemistry \& Microbiology, Korea Forest Research \\ Institute, Dongdaemun-gu, Seoul 130-712, ${ }^{4}$ School of Biotechnology and Bioengineering, Kangwon \\ National University, Chuncheon 200-701, and 5)Department of Medical Genetics and Experimental \\ Animal Center, College of Medicine, Hallym University, Chuncheon 200-702, South Korea
}

\begin{abstract}
In this study, we focused on age-dependent changes in intracellular iron deposition in the gerbil hippocampus. At 1 month of age (PM 1), iron reactivity was weak in the gerbil hippocampus. At this time, cells in the polymorphic layer of the dentate gyrus showed weak iron reactivity. At PM 3, iron reactivity in cells had not changed significantly. Thereafter, iron reactivity in the CA1-3 regions and in the dentate gyrus increased with time until PM 18. At PM 24, iron reactivity in all the subfields was similar to that at PM 18. In animals aged PM 18-24, iron positive cells had various shapes, and had processes which contained iron. These results suggest that the increase of iron deposition may be associated with normal aging and that the iron deposition in the aged hippocampus is different according to hippocampal subfields.
\end{abstract}

Key words: aging, gerbil, hippocampus, iron, oligodendrocytes

\section{Introduction}

Mongolian gerbils are genetically homogeneous and easy to handle. In addition, they have a relatively short lifespan. They are also similar to humans in several ways that make them particularly useful as a model of epilepsy, stroke, auditory processes, and in several behavioral paradigms $[5,6,9,10,13]$. It is easy to induce a transient forebrain ischemia in gerbils and they are useful for neuroscience research, especially, for the study of neurodegeneration. Aging can be defined as the nonfunctional alteration of structure or homeostatic capability in an individual organism as it passes through its lifespan [3].

Considerable attention has been directed towards understanding the biological functions of glia in the central nervous system (CNS). Glia are divided into two families: macroglia and microglia. The microglia are a group of small-sized cells of mesodermal origin, while the macroglia are a group of large-sized cells of ectodermal origin. The latter is further divided into astrocytes and oligodendrocytes [18]. In the normal state of the brain, active forms of oligodendrocytes are detected, but microglia and astrocytes are not. Agedependent changes of astrocytes and microglia have been reported in many studies $[11,12,14,19]$; how-

(Received 13 April 2006 / Accepted 31 August 2006)

Address corresponding: M.H. Won, Department of Anatomy, College of Medicine, Hallym University, Chunchon 200-702, South Korea 
ever age-dependent changes of oligodendrocytes have been relatively less investigated [1]. Moreover, there is no report of age-dependent changes of iron deposition in the hippocampus of the gerbil.

Oligodendrocytes are associated with myelination in neurons in the CNS, and they have the highest iron content in brain cells [4]. Oligodendrocytes may be more vulnerable than other cells to the release of iron. In addition, since they store the highest iron content, their particular subunit composition makes their iron content available with relatively greater ease than other cells [2]. Therefore, in this study, we focused on agedependent changes in iron positive cells and their iron reactivity in the hippocampus of gerbils.

\section{Materials and Methods}

\section{Experimental animals}

We used the male progeny of Mongolian gerbils (Meriones unguiculatus) obtained from the Experimental Animal Center, Hallym University, Chunchon, South Korea. At 1 month, 3, 6, 12, 18 and 24 months of age (PM 1, PM 3, PM 6, PM 12, PM 18 and PM 24, respectively), 7, 7, 7, 5, 5 and 5 gerbils, respectively, were sacrificed for the experiment. The animals were kept in an air-conditioned room at a temperature of $23^{\circ} \mathrm{C}$ and a humidity of $60 \%$, and lights were on $12 \mathrm{~h} \mathrm{a}$ day from 6:00 to 20:00. The animals were provided with free access to water and diet containing crude protein $(20 \%$ of diet) (Cheil Jedang, South Korea). Procedures involving animals and their care conformed to the institutional guidelines which are in compliance with current international laws and policies (NIH Guide for the Care and Use of Laboratory Animals, NIH Publication No. 85-23, 1985, revised 1996) and were approved by the Hallym's Medical Center Institutional Animal Care and Use Committee. All experiments were conducted to minimize the number of animals used and suffering caused.

\section{Tissue processing}

Animals were sacrificed under deep anesthesia by injecting sodium pentobarbital $(40 \mathrm{mg} / \mathrm{kg}$ body weight, I.P.) and transcardial perfusion with $0.1 \mathrm{M}$ phosphatebuffered saline (PBS, pH 7.4) containing $0.1 \%$ sodium nitrite and $1 \mathrm{U} / 100 \mathrm{ml}$ of heparin, followed by $4 \%$ paraformaldehyde in $0.1 \mathrm{M}$ PBS. After perfusion, fore- brains with the hippocampus were removed, post-fixed for $4-6 \mathrm{~h}$ in the same fixative at $4^{\circ} \mathrm{C}$, and stored in a $30 \%$ phosphate-buffered sucrose solution for 12-24 h. Thereafter the frozen tissues were serially sectioned transversely at $30 \mu \mathrm{m}$ thickness with a cryostat and then collected into six-well plates containing PBS [8].

\section{Histochemistry for iron}

The sections were immersed in Perl's solution (1:1, $2 \% \mathrm{HCl}$ and $2 \%$ potassium ferrocyanide) at room temperature for $30 \mathrm{~min}$, rinsed in deionized water for 30 min, then immersed for $15 \mathrm{~min}$ in filtrated $0.5 \% \mathrm{DAB}$ in PBS ( $\mathrm{pH}$ 7.4) according to a previously described method [7]. One milliliter 1 of $1 \%$ hydrogen peroxide was then added for every $200 \mathrm{ml}$ of DAB solution. The tissue remained in this solution for $15 \mathrm{~min}$ and was then rinsed in deionized water for at least $30 \mathrm{~min}$. Sections were mounted with Canada Balsam (Kanto, Japan). For negative control, endogenous peroxidase activity was determined by processing representative sections in DAB without pretreatment with Perl's reaction. All staining procedures were conducted within $24 \mathrm{~h}$ after sectioning.

\section{Quantification of data and statistical analysis}

All measurements were performed, in order to ensure objectivity, under blind condition by two observers carrying out the measures of experimental sections under the same condition in each experiment. For the quantitative analysis of iron reactivity in the hippocampus, 15 sections per animal were randomly selected within the corresponding levels of the hippocampus.

The corresponding areas of hippocampal subfields were measured on a monitor at a magnification of $25-$ 50 times. Images of all iron positive structures taken from 3 layers (strata oriens, pyramidale and radiatum in the hippocampus proper, and molecular, granule cell and polymorphic layers in the dentate gyrus) were obtained through an Axiophot light microscope (Carl Zeiss, Germany) connected via a CCD (Charge Coupled Device) camera to a PC monitor. Video images were digitized into an array of $512 \times 512$ pixels corresponding to a tissue area of $140 \times 140 \mu \mathrm{m}$ (40× primary magnification). Each pixel had a resolution of 256 gray levels. The staining intensity was evaluated by means of a relative optical density (ROD) value, which was obtained after transformation of mean gray values 
using the formula: $\mathrm{ROD}=\log (256 /$ mean gray value $)$. We measured the ROD of the complete field, and the values of the background staining were subtracted from the ROD values of all reactive structures before statistical processing. The relative $\%$ of control levels were plotted on a graph.

Inter-animal differences in each group, as well as interexperimental differences, were not statistically significant. The values presented represent the means of experiments performed for each hippocampal area. Differences among the means were statistically analyzed by one-way analysis of variance followed by Duncan's new multiple range method or the Newman-Keuls test to elucidate the age-related changes of iron reactivity or to investigate the differences between PM 1 and other age groups. $P<0.05$ was considered significant.

\section{Results}

\section{CAl region}

In the PM 1 age group, iron reactivity in the hippocampal CA1 region was very weak (Fig. 1A). Iron reactive cells were detected in strata oriens, pyramidale and radiatum and their iron reactive cells were round in shape. At PM 3, iron deposition had slightly increased compared to that of the PM 1 group (Figs. 1B and 4A). In the PM 6 group, iron deposition had increased compared to that of the PM 3 group (Figs. 1C and 4A). In the PM 12 group, iron deposition was significantly increased in the hippocampal CA1 region compared to the PM 6 group (Figs. 1D and 4A). At this age, the stratum pyramidale showed strong iron reactivity. In the PM 18 group, iron deposition was significantly increased in the hippocampal CA1 region compared to the PM 12 group (Figs. 1E and 4A). In this age group, iron reactive cells were multiform and had well-stained processes which projected in various directions. At PM 24, iron deposition in the CA1 region was similar to that seen in the PM 18 age group (Figs. 1F and 4A).

\section{CA2/3 region}

Iron reactivity in iron positive cells in the hippocampal CA2/3 region was very weak in the PM 1 age group (Fig. 2A). The pattern of change in iron deposition from PM 3 to PM 6 was similar to that in the CA1 region (Figs. 2B-2C and 4B). In the PM 12 age group, iron deposition increased in the hippocampal CA2 re- gion, while in the hippocampal CA3 region, iron reactivity was not altered (Fig. 2D). In the PM 18 age group, iron deposition was significantly increased in the hippocampal CA2/3 region compared to the PM 6 age group (Figs. 2E and 4B). In this age group, round form iron reactive cells were detected in the stratum oriens of the hippocampal CA2/3 region. In the PM 24 age group, iron deposition was similar to that seen in the PM 18 age group (Figs. 2F and 4B).

\section{Dentate gyrus}

The pattern of change in iron deposition in the dentate gyrus was similar to that in the hippocampal CA2/ 3 region (Figs. 3 and 4B). In the PM 3 and PM 6 age groups, iron deposition was similar to that in the PM 1 age group (Figs. 3A-C and 4C). In the PM 12 age group, iron deposition was significantly increased in the granule cell layer and polymorphic layer of the dentate gyrus compared to the PM 1 age group (Figs. 3D and 4C). In this group, iron deposition was significantly increased in the suprablade of the dentate gyrus compared to that in the infrablade of the dentate gyrus (Fig. 3D). Iron deposition further increased in the dentate gyrus in the PM 18 age group (Figs. 3E and 4C). In the PM 24 age group, iron deposition in the dentate gyrus was similar to that in the PM 18 age group (Figs. $3 \mathrm{~F}$ and $4 \mathrm{C})$.

\section{Discussion}

Many studies have investigated age-related changes in microglia and astrocytes in brains of rodents, nonhuman primates and humans. Microglia-associated antigens increase with age [16, 19,20], and glial fibrillary acidic protein increases in astrocytes $[11,12,14]$. However, very few studies have reported on age-related changes of oligodendrocytes and iron reactivity in the hippocampus.

In the present study, we observed that iron deposition in iron positive cells in the gerbil hippocampus began to increase significantly at PM 6, and iron deposition was highest at PM 18. This result is similar to that of a previous study which showed the iron level in the aged rat brain was significantly elevated [1].

We also found in the present study that iron reactivity in the aged gerbil hippocampus was shown in processes of iron positive cells, and that the morphol- 

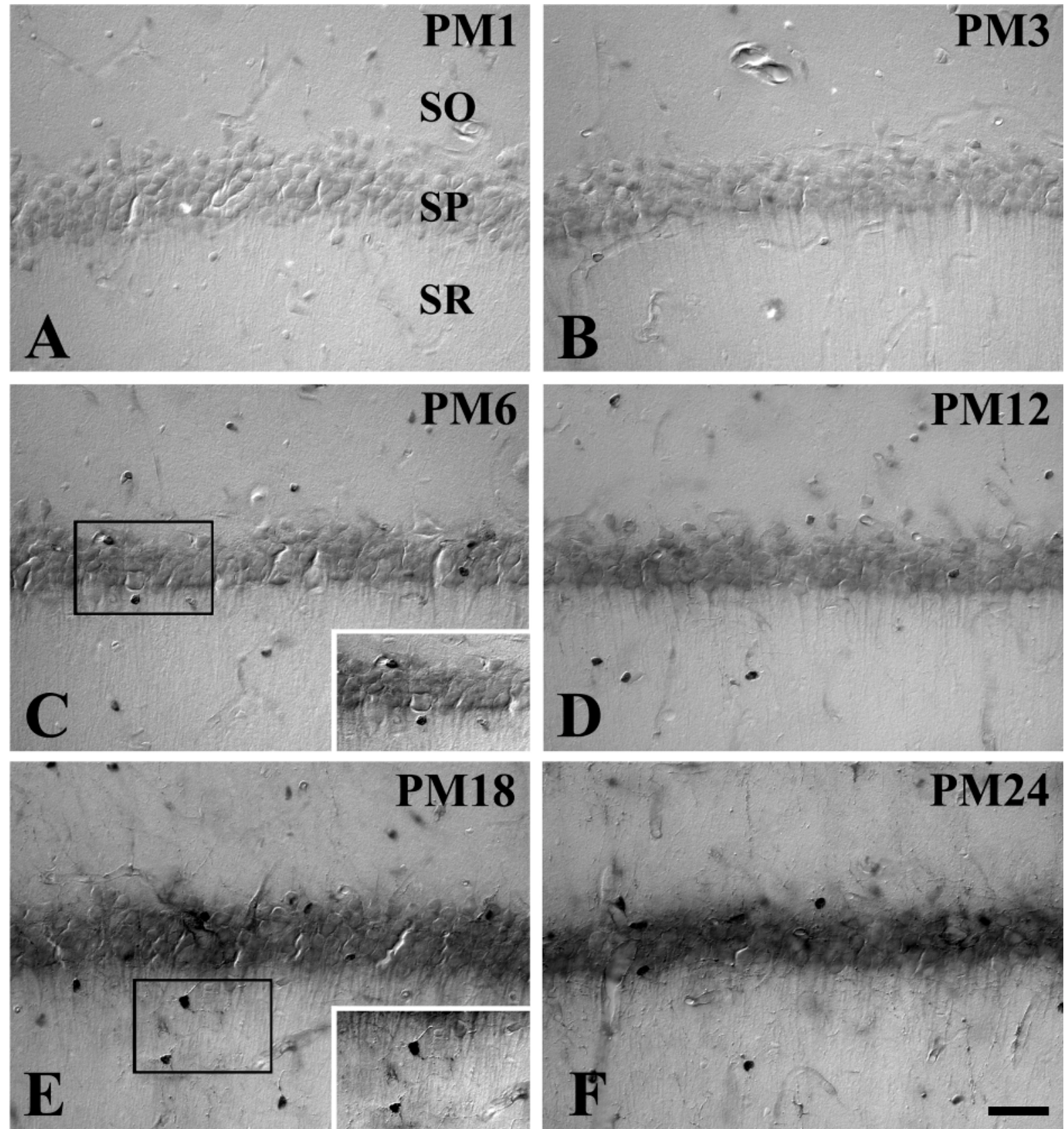

Fig. 1. Histochemical staining for iron in the CA1 region at PM 1 (A), PM 3 (B), PM 6 (C), PM 12 (D), PM 18 (E) and PM 24 (F). At PM 3, iron reactivity in iron positive cells is higher than that in the PM1 group. Thereafter iron deposition in iron positive cells increases with time until PM 18. Iron reactivity at PM 24 is similar to that at PM 18 (F). Iron positive processes are apparent in the strata oriens (SO) and radiatum (SR) at PM 18 (E). SP, stratum pyramidale. Bar $=50 \mu \mathrm{m}$.

ogy of iron positive cells became various in the hippocampus with age. Oligodendrocytes are present in the white and grey matter of the CNS. It has been reported that oligodendrocytes constitute a heterogeneous group, in which 3 types of cell have been distinguished: those of light, medium and dark cytoplasm [23]. The author of that study reported that the number of light oligodendrocytes decreased with age, while the number of dark oligodendrocytes increased. In our study, we found that iron reactivity in iron positive cells increased in 

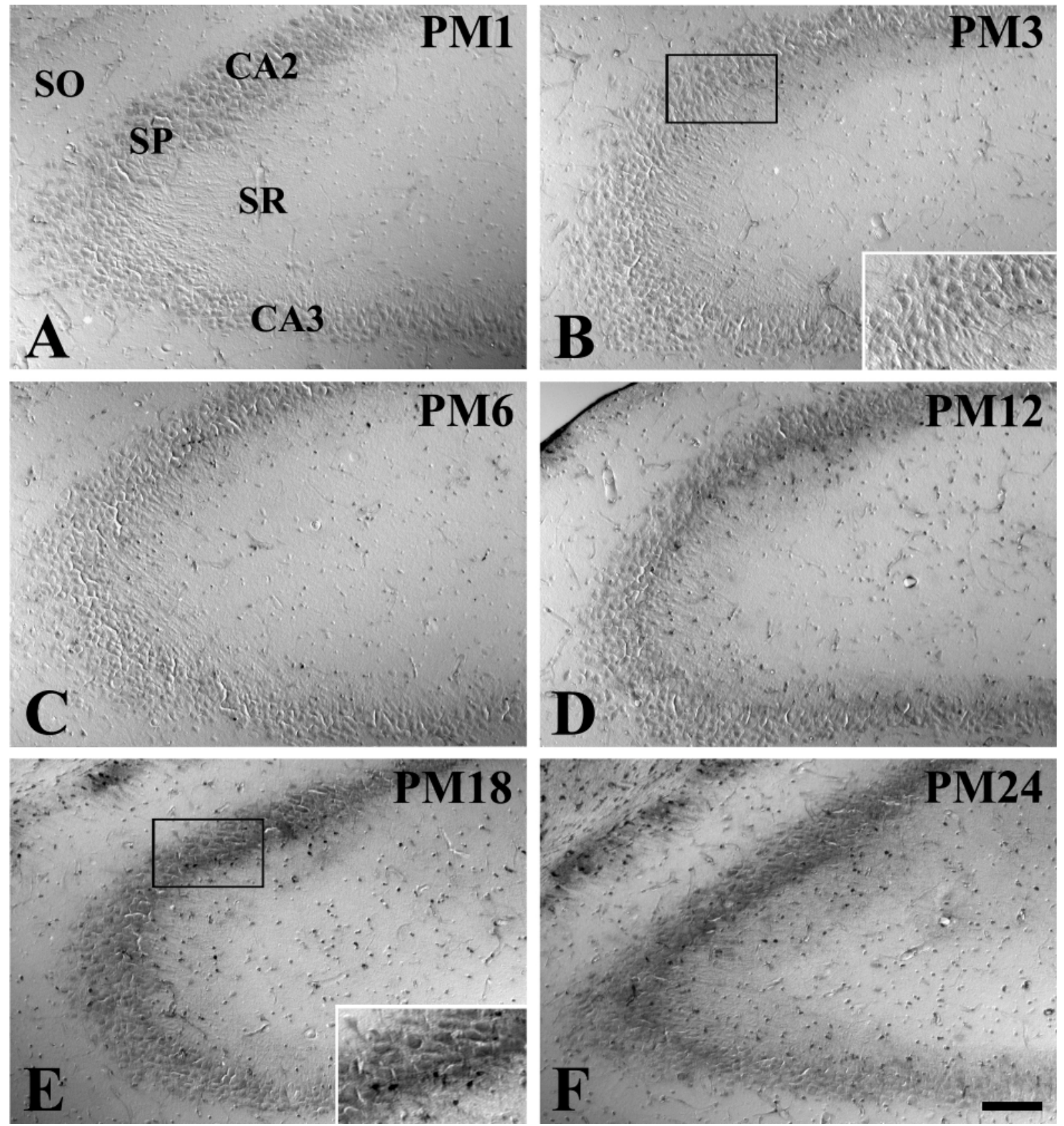

Fig. 2. Histochemical staining for iron in the CA2/3 region at PM 1 (A), PM 3 (B), PM 6 (C), PM 12 (D), PM 18 (E) and PM 24 (F). Iron reactivity increases significantly in the PM 18 age group compared to the PM 1 age group. SO, stratum oriens; SP, stratum pyramidale; SR, stratum radiatum. Bar $=100 \mu \mathrm{m}$.

the hippocampus with age.

Oligodendrocytes are associated with myelination in the CNS and normally have the highest iron content among the brain cells, containing as much as $70 \%$ of the brain iron [4]. Damage to oligodendrocytes could increase the iron content in the damaged areas. An increase of iron in damaged areas created hydroxyl radicals via reaction with superoxide radicals [21, 24]. Hence, the age-related loss/dysfunction of myelin would result in a further increase in the production of damaging free radicals. Reactive oxygen species-induced lipid peroxidation could predispose myelin to breakdown and 

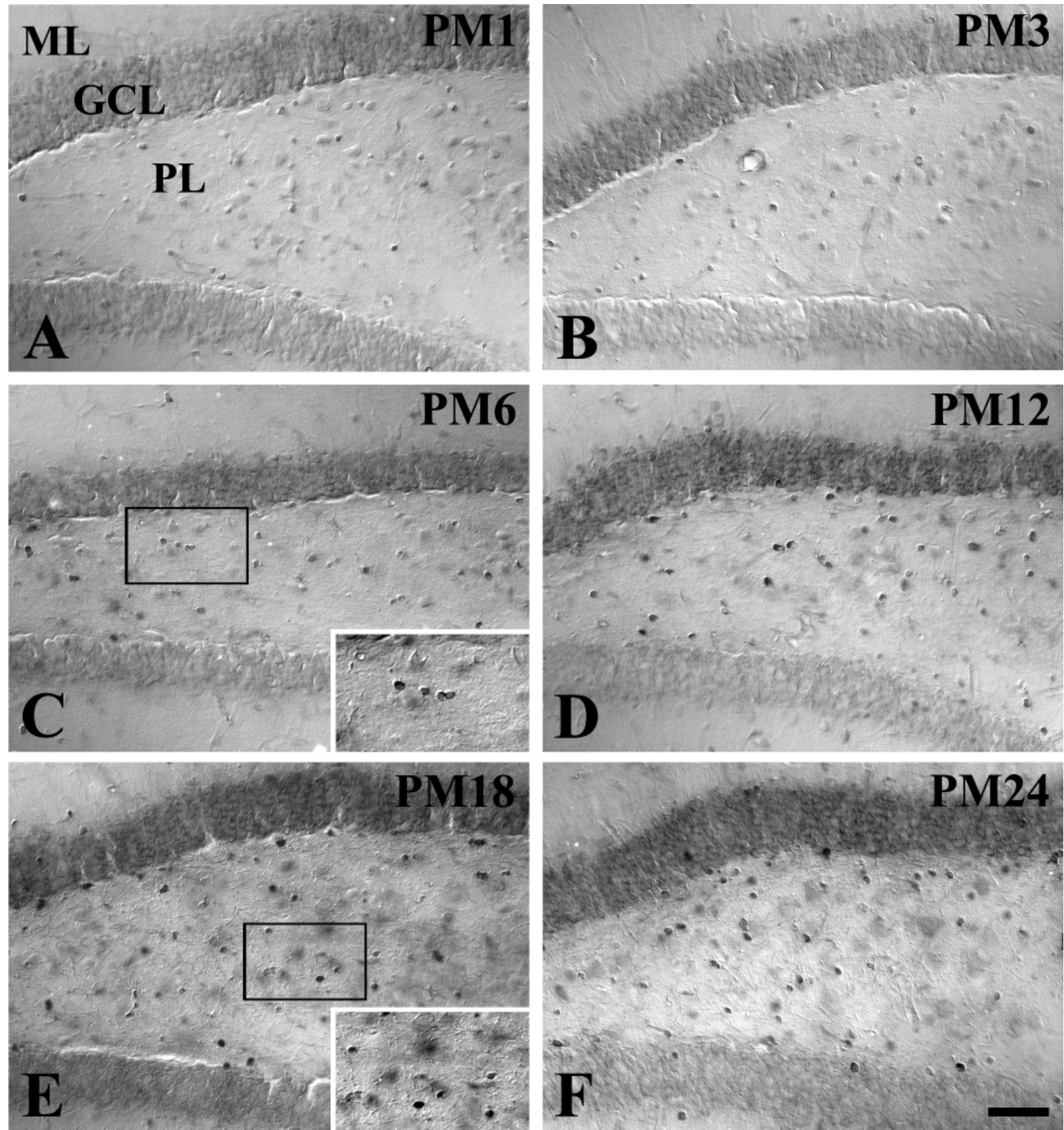

Fig. 3. Histochemical staining for iron in the dentate gyrus at PM 1 (A), PM 3 (B), PM 6 (C), PM 12 (D), PM 18 (E) and PM 24 (F). At PM 3, iron reactivity is similar to that in the PM 1 age group. Thereafter iron deposition increases with time until PM 18. Iron reactivity at PM 24 is slightly lower than that at PM 18. GCL, granule cell layer; ML, molecular layer; PL, polymorphic layer. Bar $=50 \mu \mathrm{m}$.

possibly degrade its electrical insulation properties that are necessary for saltatory conduction $[17,22]$. Myelin damage or loss can contribute to this process as it has a pronounced effect on neuronal survival and function through a variety of mechanisms.
Several observations suggest that iron is an essential factor in myelination and oligodendrocyte biology. However, the specific role of iron in these processes remains to be elucidated. Its role could be an essential cofactor in metabolic processes or a transcriptional or 


\section{(A) CAI}

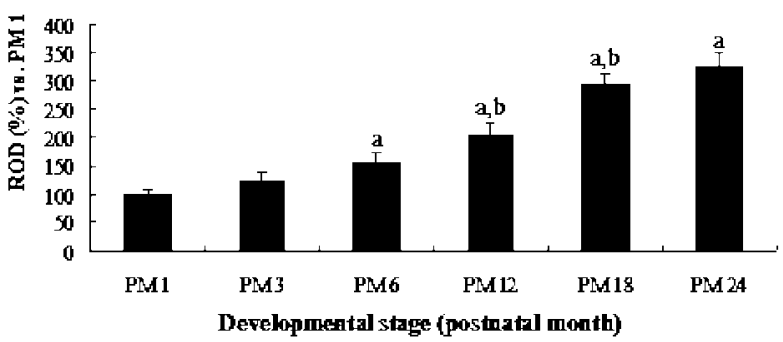

\section{(B) $\mathrm{CA2} / 3$}

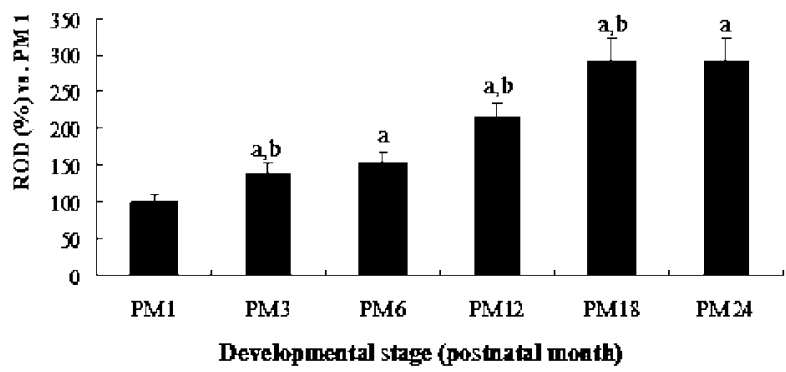

(C) Dentate gyr 1 s

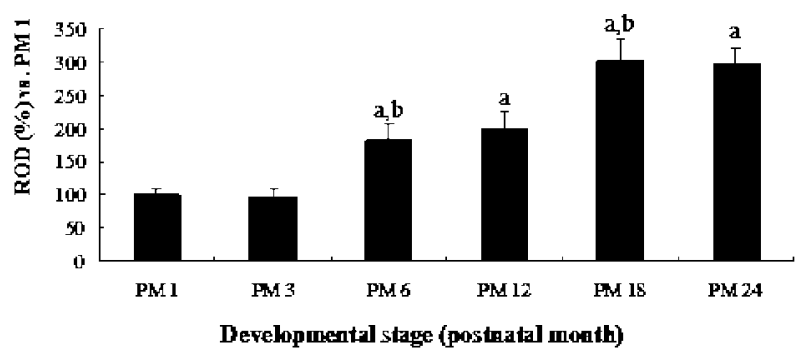

Fig. 4. The ROD of iron reactivity in the hippocampus proper and the dentate gyrus at various ages. The results of the quantitative data obtained using image analysis are consistent with the histochemical data $\left({ }^{\mathrm{a}} P<0.05\right.$, significantly different from the PM1 age group, ${ }^{\mathrm{b}} P<0.05$, significantly different from the pre-adjacent group). The bars indicate the means \pm S.D.

translational regulator [15]. In conclusion, our study indicates that iron deposition in the gerbil hippocampus increases with age and this increase may be associated with neurodegeneration in aged brains.

\section{Acknowledgement}

The authors would like to thank Mr. Seok Han, Mr. Seung Uk Lee and Ms. Hyun Sook Kim for their technical help in this study. This work was supported by the Research Innovation Center at Hallym University.

\section{References}

1. Benkovic, S.A. and Connor, J.R. 1993. Ferritin, transferrin, and iron in selected regions of the adult and aged rat brain. J. Comp. Neurol. 338: 97-113.

2. Blissman, G., Menzies, S., Beard, J., Palmer, C., and Connor, J. 1996. The expression of ferritin subunits and iron in oligodendrocytes in neonatal porcine brains. Dev. Neurosci. 18: 274-281.

3. Cristofalo, V.J. 1988. Cellular biomarkers of aging. Exp. Gerontol. 23: 297-307.

4. de los Monteros, A.E., Korsak, R.A., Tran, T., Vu, D., de Vellis, J., and Edmond, J. 2000. Dietary iron and the integrity of the developing rat brain: a study with the artificially-treated rat pup. Cell Mol. Biol. 46: 501-515.

5. Hafidi, A., Lanjun, G., and Sanes, D.H. 1999. Age-dependent failure of axon regeneration in organotypic culture of gerbil auditory midbrain. J. Neurobiol. 41: 267-280.

6. He, D.Z. and Dallos, P. 1997. Expression of potassium channels in gerbil outer hair cells during development does not require neural induction. Dev. Brain Res. 103: 95-97.

7. Hill, J.M. and Switzer, RC 3rd. 1984. The regional distribution and cellular localization of iron in the rat brain. Neuroscience 11: 595-603.

8. Hwang, I.K., Kim, D.W., Yoo, K.Y., Kim, D.S., Kim, K.S., Kang, J.H., Choi, S.Y., Kim, Y.S., Kang, T.C., and Won, M.H. 2004. Age-related changes of gamma-aminobutyric acid transaminase immunoreactivity in the hippocampus and dentate gyrus of the Mongolian gerbil. Brain Res. 1017: 77-84.

9. Hwang, I.K., Yoo, K.Y., Kim, D.S., Eum, W.S., Park, J.K., Park, J., Kwon, O.S., Kang, T.C., Choi, S.Y., and Won, M.H. 2004. Changes of pyridoxal kinase expression and activity in the gerbil hippocampus following transient forebrain ischemia. Neuroscience 128: 511-518.

10. Hwang, I.K., Yoo, K.Y., Park, J.K., Nam, Y.S., Lee, I.S., Kang, J.H., Choi, S.Y., Lee, J.Y., Kang, T.C., and Won, M.H. 2004. Ischemia-related changes of adrenocorticotropic hormone immunoreactivity and its protective effect in the gerbil hippocampus after transient forebrain ischemia. Neuroscience 126: 871-877.

11. Kohama, S.G., Goss, J.R., Finch, C.E., and McNeill, T.H. 1995. Increases of glial fibrillary acidic protein in the aging female mouse brain. Neurobiol. Aging 16: 59-67.

12. Laping, N.J., Teter, B., Nichols, N.R., Rozovsky, I., and Finch, C.E. 1994. Glial fibrillary acidic protein: regulation by hormones, cytokines, and growth factors. Brain Pathol. 4: 259-275.

13. Lee, H.Y., Hwang, I.K., Kim, D.H., Kim, J.H., Kim, C.H., Lim, B.O., Kang, T.C., Bang, K.H., Seong, N.S., Lee, H.J., Kim, J.D., and Won, M.H. 2005. Ischemia-related changes in galanin expression in the dentate hilar region after transient forebrain ischemia in gerbils. Exp. Anim. 54: 21-27.

14. O'Callaghan, J.P. and Miller, D.B. 1991. The concentration of glial fibrillary acidic protein increases with age in the 
mouse and rat brain. Neurobiol. Aging 12: 171-174.

15. Ortiz, E., Pasquini, J.M., Thompson, K., Felt, B., Butkus, G., Beard, J., and Connor, J.R. 2004. Effect of manipulation of iron storage, transport, or availability on myelin composition and brain iron content in three different animal models. J. Neurosci. Res. 77: 681-689.

16. Perry, V.H., Matysak, M.K., and Fearn, S. 1993. Altered antigen expression of microglia in the aged rodent CNS. Glia 7: 60-67.

17. Peters, A. and Sethares, C. 2002. Aging and the myelinated fibers in prefrontal cortex and corpus callosum of the monkey. J. Comp. Neurol. 442: 277-291.

18. Rezaie, P. and Male, D. 2002. Mesoglia \& microglia - a historical review of the concept of mononuclear phagocytes within the central nervous system. J. Hist. Neurosci. 11: 325-374.

19. Rozovsky, I., Finch, C.E., and Morgan, T.E. 1998. Agerelated activation of microglia and astrocytes: in vitro studies show persistent phenotypes of aging, increased proliferation, and resistance to down-regulation. Neurobiol. Aging 19: 97-103.
20. Sheffield, L. and Berman, N.E. 1998. Microglial expression of MHC class II increases in normal aging of non-human primates. Neurobiol. Aging 19: 47-55.

21. Smith, M.A., Harris, P.L., Sayre, L.M., and Perry, G. 1997. Iron accumulation in Alzheimer disease is a source of redox-generated free radicals. Proc. Natl. Acad. Sci. USA 94: 9866-9868.

22. Surewicz, W.K., Epand, R.M., Epand, R.F., Halett, F.R., and Moscarello, M.A. 1986. Modulation of myelin basic protein-induced aggregation and fusion of liposomes by cholesterol, aliphatic aldehydes and alkanes. Biochim. Biophys. Acta 863: 45-52.

23. Wawrzyniak-Gacek, A. 2002. Distribution of various types of oligodendrocytes and cellular localisation of iron in the frontal cortex of the adult rat. Folia. Morphol. (Warsz) 61: $115-121$.

24. Xie, C.X., St Pyrek, J., Porter, W.H., and Yokel, R.A. 1995. Hydroxyl radical generation in rat brain is initiated by iron but not aluminum, as determined by microdialysis with salicylate trapping and GC-MS analysis. Neurotoxicology 16: 489-496. 